\title{
Imaginary Versus Virtual Loci: Evaluating the Memorization Accuracy in a Virtual Memory Palace
}

\author{
Jan-Paul Huttner \\ Technische Universität \\ Braunschweig \\ j-p.huttner@tu-bs.de
}

\author{
David Pfeiffer \\ Technische Universität \\ Braunschweig \\ d.pfeiffer@tu-bs.de
}

\author{
Susanne Robra-Bissantz \\ Technische Universität \\ Braunschweig \\ s.robra-bissantz@tu-bs.de
}

\begin{abstract}
The Method of Loci (MOL) is a mnemonic strategy to memorize declarative knowledge. In the late $90 \mathrm{~s}$ researchers started to experimentally analyze the MOL by providing their participants a virtual world. This digital environment was meant to serve the users as a template for their personal memory palace. Past studies differed in central aspects of their experimental design. One difference is that earlier studies integrated the learning content in multiple and different ways. The design of the learning content is an essential aspect and should make a difference based on cognitive theories. This study analyzes if participants perform better with virtually presented learning content or if the MOL works better when users have to imagine the content. Results encourage future concepts of the virtual MOL to implement the learning content in the environment.
\end{abstract}

\section{Introduction}

This study addresses researchers and practitioners in the field of human-computer interaction, especially in an educational context.

The Method of Loci (MOL), also called memory palace, belongs to the set of mnemonic techniques. These techniques rely on the principle to memorize the learning content by mentally associating it with another entity. For instance, this entity could be an abbreviation or an image. Even a mental map of a spatial environment might serve the user as an association anchor [30]. According to Yates (1999), this way of memorizing information makes it easier for the user to recall them [41]. However, when choosing these entities one should consider the aspect of cognitive load to avoid the negative effects of overload [25]. In the context of education, mnemonic strategies can be used to master the first level of learning, "remembering", which is described by Krathwohls' revised taxonomy of learning domains, originally proposed by Bloom [15].

Hartwig and Dunlosky (2012) as well as McCabe (2011) emphasized that mnemonic strategies should be integrated in the students' curriculum, as they may foster their chances to successfully perform in college [9][26]. Moreover, Putnam (2015) illustrated a positive correlation between the students' motivation to learn and the use of mnemonic strategies. He pointed out that learning of basic principles by the help of mnemonics results in more time to cope with higher order learning. The approach to teach and use these strategies in the students' careers was already promoted in 1990 by Levin and Levin [17]. For instance, they conducted a study designed for an educational application. In an experiment, authors showed that students that used a set of so-called mnemonomies outperformed a control group that had to memorize only botanical terms. In this case, mnemonomies were pictorial compositions made to illustrate hierarchical relationships within a botany classification system. However, as stated by Putnam (2015), mnemonic methods were not fully implemented in the teaching community.

\subsection{Method of Loci}

The MOL, which is the key concept investigated in this study, is a mnemonic strategy that helps the user to memorize declarative knowledge, e.g. lists of words, numbers or facts. It is based on the approach to facilitate the memorization process by building a mental association between the learning content and spatial cues. As a simplified example, if one intends to memorize a list of items like pencil, apple, chair, fork and cup the user mentally walks through a well-known environment, like his apartment, and puts these items at certain places (place $=$ lat. locus, pl. loci). For instance, one could lay the fork on top of the kitchen table and place the apple into the stove. Later, if these 
items shall be recalled, the user again walks through the apartment in his or her mind and can easily remember the visualized fork on the table or the apple in the stove.

However, the training phase needed to handle the MOL, especially for more complex tasks, might be mentally tiring. Authors reported training phases between two hours and several days [4, 33].

The MOL was and is still investigated from many point of views, not only from an educational perspective. Neuroscientists are also researching on this strategy and trying to understand the neural mechanism lying behind it $[6,20]$.

\subsection{Virtual Memory Palaces}

Researchers in the field of computer science and psychology started to investigate the potential lying in virtual worlds to serve a learner as a template and support for the mental representation of the memory palace. This is the concept that will be referred to as a virtual memory palace (VMP) in the following sections. The approach illustrates a promising way for the application of the MOL in an e-learning context, especially in the domain of virtual reality (VR) research and its use for an educational purpose. Jund et al. (2016) outlined the importance of VR technology and their use for VMPs in the future [13].

\section{Related Work}

Within the last years, several studies analyzed different aspects of the MOL in a virtual context. Researchers aimed to answer a variety of research questions, e.g. whether the virtual MOL outperforms the traditional MOL, if the vMOL leverages the users' long term memory or how the vMOL can be applied to an augmented reality context. In the following, a brief description of related work will be given to illustrate the theoretical base and motivation that leads to this research approach (see section 3 ).

In 1997 Storkerson and Wong introduced the idea of enhancing mnemonic strategies, like the MOL, with the help of hypermedia. A higher level of intelligibility of communications would foster the memorization process in a spatial context [40]. Later in the year 2000, Hedman and Bäckström created a VMP that was designed to help students in a philosophy course [10]. Its architecture was strongly inspired by a traditional museum but even though authors vaguely emphasize the importance of key principles for designing a VMP, it did not follow any specific rules. The loci basically consisted of text, written on the virtual walls. The corresponding experiment did not show any effects that imply a superiority of the VMP over a traditional learning technique. Still, it is noteworthy that participants suggested building interactive 3D loci. Furthermore, authors observed that the distance between rooms and loci may lead to navigational problems for the students [10]. Hence, a need for further research of design principles of a VMP is implied at this point.

Six years later, Fassbender and Heiden followed a similar idea and hypothesized that exploring a VMP facilitates the MOL as the user does not have to stress his or her mind to create a completely mentally based representation of the environment [7]. They also conducted an experiment. The design of their VMP was inspired by a traditional castle of medieval times. The loci were integrated in the environment, animated and enriched with sound features. They argued that these extra cues should foster the participants' mental associations. As a result, authors found that the longterm memory improved due to the MOL treatment. Their implementation of the VMP allowed the users to self-select the loci and to put the to-be-remembered items in context. Moreover, they described six (unreferenced) rules that should be respected when building a VMP, e.g. that the loci should be put in places that are not too spacious or too narrow. Similar to Hedman and Bäckström, they emphasize the importance of a unique and unusual design of the loci. Nevertheless, the descriptions are quite vague and the theoretical base seems incomplete. Again, the need for deeper investigation is seen here.

Legge et al. published their work in 2012 [16]. They analyzed three groups with a total of 142 students to reveal possible advantages of a virtual MOL protocol over the traditional method. Their study design included two experimental groups (traditional and virtual MOL) and a control group (no specific learning strategy). The control group was outperformed by the MOL groups while the MOL groups did not show significant differences. Still, the authors could show that the recall accuracy between three different virtual environments (apartment, warehouse and school) within the vMOL group did not differ. This indicates that the environments' influence on the memorization and recall performance is not too important. However, their VMP and experimental design differ from other studies in a central aspect. Legge et al. (2012) did not implement any loci in their VMPs. The items that the participants had to memorize were presented after an exploration phase of five minutes in the virtual environments. This is a different approach compared to the other studies that integrated their loci in different ways. Hence, participants probably had to invest more mental effort to imagine the items and associate them with the loci. 
Jund et al. (2016) evaluated two different frames of reference when users apply the MOL in a virtual environment. In an experiment, they used two groups to gather data about the participants recall performance, depending on the frame of reference. They found out that an egocentric frame outperforms an allocentric condition to offer the participants' spatial cues for the memorization process. Their loci were designed as images of colorful textures and patterns to avoid a semantic bias in the memorization process of the participants.

Another investigation was performed by Mann et al. (2017)[23]. In a pilot study the authors compared the memorization and recall accuracy between four groups including a control group. One group was instructed to use the traditional MOL and two groups used a VMP. The two VMP groups explored the virtual environment either on a desktop computer or in a Cave Automatic Virtual Environment (CAVE). This is basically a small room in which all four walls serve as a projection surface for the virtual environment. Each locus consisted of a 3-dimensional object and a descriptive text (the word to be remembered). As expected, results indicated a superiority of the vMOL groups over the control and the traditional MOL group.

As mentioned earlier, all studies investigated different research questions. However, the theoretical base for the design of the environment and loci is not fully evaluated.

\section{Theory \& Research Approach}

As described in the introduction, this study aims to contribute to design guidelines for VMPs in practice. Driven by this intention, the design science methodology (Hevner 2004) [12], more precisely, the process described by Peffers (2007) was chosen as a framework for the following study [29]. Peffers' research process consists of six essential phases including the definition of a problem, followed by the theoretical base for a better solution, an artefact plus its demonstration and evaluation and finally the communication of the findings. Section one and two sought to outline the problem and show the potential lying in the VMP for an educational purpose. Consequently, we will describe the research question including its necessity and derive appropriate hypotheses. Section four contains the description of the artefact and the experimental design for the evaluation. The summary and discussion of the findings will be covered in the last sections.

The variety of VMP concepts was shown briefly in section two. Focusing on the loci in this study, table 1 summarizes the different design features that were implemented by related experiments and concepts.

Table 1. Overview loci features

\begin{tabular}{|c|c|c|c|c|c|c|}
\hline & $3 \mathrm{D}$ & Image & Text & External & Animated & Audio \\
\hline $\begin{array}{l}\text { Hedman \& } \\
\text { Bäckström }\end{array}$ & & & $\mathrm{x}$ & & & \\
\hline Mann et al. & $x$ & & $x$ & & & \\
\hline Jund et al. & & $x$ & & & & \\
\hline $\begin{array}{l}\text { Fassbender \& } \\
\text { Heiden }\end{array}$ & $x$ & $x$ & & & $x$ & $x$ \\
\hline Legge et al. & & & $x$ & $x$ & & \\
\hline
\end{tabular}

Clearly, the loci design of past studies varies substantially. This might be due to the fact that, besides others, the traditional MOL is performed completely in mind. Earlier studies that transferred the MOL into a virtual world context did not explicitly evaluate to which degree the method should be performed in mind, especially regarding the conceptualization and design of loci. Since the traditional MOL heavily relies on the idea to mentally establish an individual, unusual and unique visual association between an item and a locus, it seems questionable whether a predefined and integrated locus is better suited for the memorization process than a completely mind-based locus. Therefore, this study aims to answer the research question whether in a VMP, the concept of imaginary or visualized locus leads to better user memory.

In the dual coding theory, Paivio and Lambert (1981) assert that human cognition and memory depend heavily on two connected neural systems. [28]. Despite their interconnected design, they are capable of working independently. While one system processes non-verbal information, the other one converts verbal input. However, based on the interconnection between them, a neural interpretation of one system is able to produce an association to representations in the other one. Malaga (2000) gave an example, stating that if a picture is named to a subject, certain images may appear in his/her visual mind while reading a particular word [21]. Furthermore, studies have revealed an improvement of subjects' recall performance if pictorial and lexical stimuli were used simultaneously [27]. This theory is also supported by other authors, who describe a superior memorization accuracy achieved with the help of a combination of text and images over either one of them solely [8, 36, 37].

Based on this theory, an experiment was conducted to investigate the effectiveness of imaginary versus virtual representations of the to-be-remembered items. Therefore, two prototypes of VMPs were implemented for virtual reality devices. The first one is equipped with virtual loci, the second one only offers text-based 
items so the users have to create their loci in mind. A more detailed description will be given in section four. In a first step, we aim to examine, whether the visual integration of the loci in the virtual world facilitates the association and memorization process compared to a protocol which demands the user to build the loci exclusively in mind (see Legge et al. 2012). Following the dual coding theory, a locus which is a combination of an image and a naming word should establish a stronger association than a text-only locus. Consequently, the participants' recall accuracy should be superior in the image-text group.

The measurement of the recall accuracy is based on the scores introduced by Legge et al. (2012). They used two different approaches to indicate the proportion of items a participant was able to remember. The strict score reflects how many words a participant was able to recall in order. For instance, a list consisting of the five items chair, pen, table, book and window would be presented to the subject. If s/he then enters pen, chair, table, window and cook the strict score would be 0.2 as only table was entered at the correct position. The second score, the lenient score, measures the overall proportion of words that could be recalled, ignoring the correct position. Considering the strict score example, the lenient score would be 0.8 since only the last item cook is not in the list.

Relating to the supposed superior influence of an image-text locus over a text-only condition, the following hypotheses are derived.

H1: Memorizing the items by the use of visual loci will result in a higher strict score than by the use of exclusively mental loci

H2: Memorizing the items by the use of visual loci will result in a higher lenient score than by the use of exclusively mental loci

\section{Experimental Design}

The following sections describe the experiment's central aspects, i.e. the participants, the technology that was used, the experimental procedure and the VMPs. As mentioned in the last section, the recall accuracy was measured using the strict and lenient score calculation. However, the list of words that were presented to the participants was not chosen randomly. First, according to Belleza (1981) the length of the item list for a MOL investigation should be longer than ten words, otherwise the effectiveness of the MOL would not be noticeable [2]. Hence, the list that was used in this study had a length of 40 words as suggested and applied by Ross and Lawrence (1968) [34].

Moreover, the items were chosen to lessen the mental effort for the mostly beginner level participants. Legge et al. (2012) stated in their study that words of a high concreteness, i.e. words that are easy to imagine, are easier to remember in general than those with a low concreteness. For instance, a word with a high level of concreteness would be spoon, while a term like arrogance is more abstract. Hence, the words the participants had to memorize in this experiment were all of a high concreteness. Kanske and Kotz (2010) evaluated a list of approx. 1000 German words with the help of 64 participants [14]. They rated a variety of the word norms, amongst others, the level of concreteness. The items in this experiment were taken from this list.

However, two groups were instructed to apply the vMOL in the VMPs. The loci in each VMP were thought to be memorized either as text-only or imagetext combination in a predefined condition. Section 4.2. is going to give a more detailed description of the design features.

\subsection{Technology}

As indicated by Jund et al. (2016) a higher level of immersion should positively influence the virtual MOL experience. Several studies refer to the beneficial effects that high immersive presence has on crucial factors (e.g. cognitive burden and processing, enjoyment or engagement) to successfully memorize and learn $[1,3,5,18,19,22,31,32,39]$. Immersive presence is experienced if one is perceptually in a location although one is not physically [39]. Hence, the VMPs were developed for a head-mounted display (HMD), respectively as a virtual reality environment. HMDs generate a higher immersive presence than e.g. a computer screen [32]. In addition to that, virtual reality is a promising technology for educational purposes. Especially the Google Cardboard technology offers a low-priced opportunity for the majority of the society [24]. In this concept, the smartphone's display generates a stereoscopic camera perspective which is focused by two lenses in the actual HMD. Due to this effect, the user perceives the virtual world in three dimensions. Therefore, the subjects were equipped with a HMD system that uses a smartphone as a display. To walk in the virtual environment, participants could use a gaming controller, which was connected wirelessly via Bluetooth to the HMD. Furthermore, participants were given a swivel chair so they could "look around" by turning around in a seated position as the head 
movement was tracked by the HMD and transferred into the virtual environment.

\subsection{Prototypes}

As described, navigating in the VMPs was done by using the gaming controller and the head movement. In order to ensure that participants adopted this way of walking through the VR, each participant had to pass a training level. After finishing their training, they were "transported" into the actual VMP to start the memorization phase. Not only the VMPs, but also the training levels differed between the two groups. Figure 1 shows the locus of the VMP of the image-text group $($ Tür $=$ door $)$.

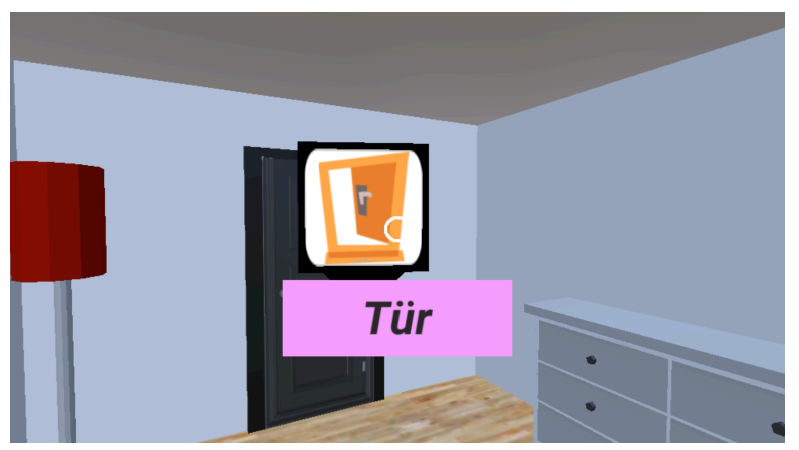

Figure 1. Locus in the image-text VMP

Both VMPs were designed as an apartment and, as stated earlier, only differed in the presentation style of the items participants had to memorize. The image-text VMP had the loci integrated at a fixed position as seen in figure 1. A fixed circle in the center of the users' field of view served as a selection tool. Participants had to look at a locus and push a button on the gaming controller to reveal the item. In the first place, each locus was only marked as a square with a question mark on it. Only then were the specific word and image uncovered. Each locus was visible for five seconds, a time frame that was adopted from Legge et al. (2012). After that, the locus disappeared. This was the time frame participants had to memorize the item at the specific locus.

In contrast to the image-text group, the text-only VMP only contained the items as a text and not fixed to a certain locus. Hence, participants had to imagine placing each item at a certain place. Again, each item was presented for five seconds and disappeared afterwards. By pushing a button on the gaming controller, the next item appeared in the users' field of vision. Note that the order of the items was the same in both conditions. The architectural design of the imagetext VMP ensured that participants had to follow a predefined walking pattern.
Summarized, the two presentation styles of the loci were directly adapted from earlier studies with the intention to evaluate, in a first step, which approach works better: the imaginary loci that were used by Legge et al. (2012) or the visually enriched loci that were integrated in the virtual worlds and implemented by other studies [7, 13, 23].

Additionally, a routine was implemented in both VMPs to measure the participants' activity in the VMP. Therefore, the system recorded the walking distance and the head rotation of the user as well as the time (seconds) $s$ /he spent in the VMP. Results and possible implications are later presented in section five.

\subsection{Participants}

In total, 60 undergraduate students, mostly majoring in technically oriented fields of study, participated in the experiment. No monetary compensation was provided. German was required to be the first language of the participants as the procedure needed them to read, understand and write lists of German words.

Due to the experimental procedure, it was possible that participants suffered from motion sickness. For instance, this effect is likely to happen if a user experiences a delay of the visual perception in the HMD compared to her/his real head movement [11]. In this case, the ability to focus on the virtual world and the level of immersive presence is weakened [39].

Nine of the students reported that due to motion sickness they were not able to concentrate on the task. Hence, these participants were removed from the data analysis leaving a total of 51 datasets (Group 1: 23, Group 2: 28). The test subjects were aged between 19 and 32 (mean $=23.73,21$ females $)$. About $11 \%$ of the participants had prior knowledge of the MOL and stated occasional use. These students were almost evenly distributed among the two groups.

\subsection{Procedure}

To avoid a self-selection bias, the participants were randomly assigned either to the image-text or the textonly group. All of the subjects took part under the same conditions, with the exception of the VMP. Figure 2 illustrates the five phases each participant had to pass. In the first phase, participants were given instructions on how to apply the vMOL. Of course, these instructions differed in terms of the description on how the items will be presented. In the following phase, participants put on the HMD and were handed the gaming controller. Before they could start the memorization phase in the VMP, the training level was loaded so the subjects could become confident with the 
navigation and handling in the virtual reality. As described in section 4.3., the virtual environment switched automatically to the VMP as soon as the participants completed the training task. In the third phase participants started to explore their specific VMP and navigated through the rooms to find and memorize the loci. Note that the memorization phase had no time limit to avoid setting the subjects under a pressure of time. The fourth phase started after the participants finished exploring all of the 40 loci in the VMP. Here, they were presented to a website that was developed to save the test subjects' input. This web interface was designed closely to the descriptions of Legge et al. (2012). Hence, it was a blank, minimalistic page containing a single text field and two buttons.

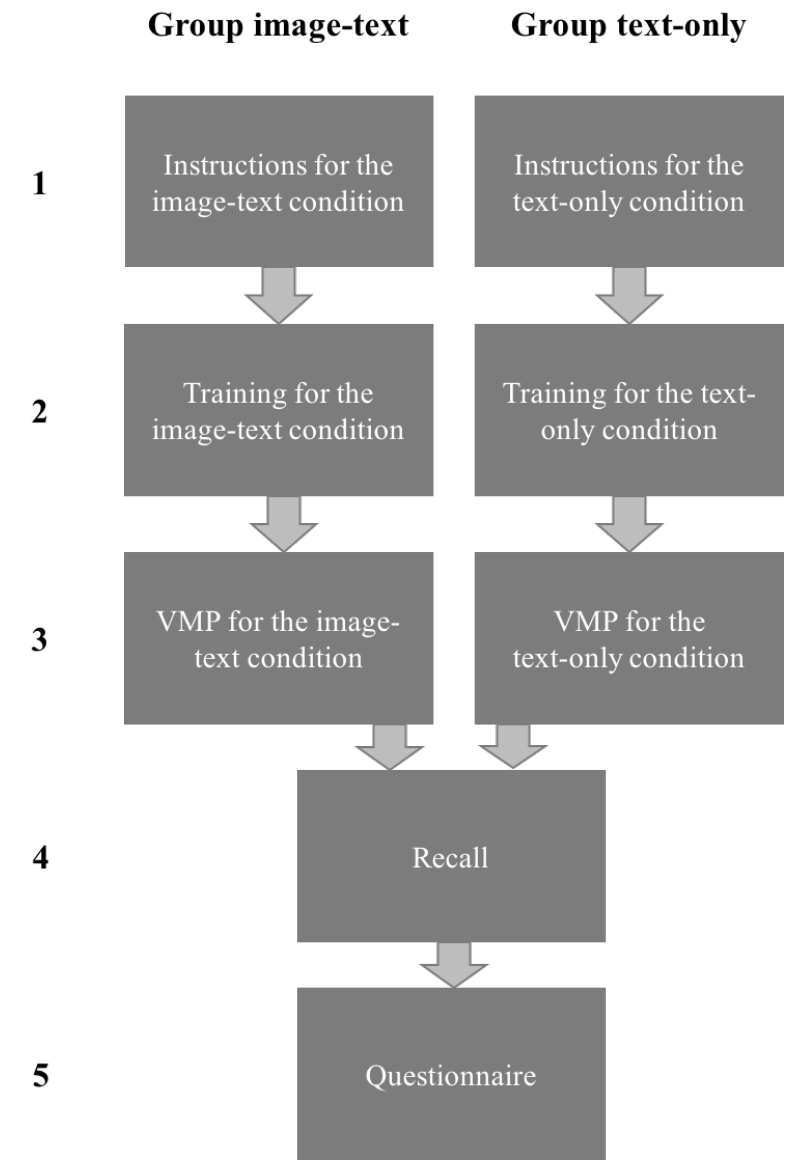

Figure 2. Experimental Procedure

One button was offered for the input confirmation, the other one was a skip button. If a participant did not know which item was next in order, s/he could simply skip the current position. Finally, in the last phase the questionnaires were handed out to aquire the sociographic information.

\section{Analysis \& Results}

In order to prepare the data for the statistical analysis, the following revision was performed. As described earlier, the scores were calculated by the amount and order of words the participants were able to recall. If necessary, these words were corrected afterwards in cases of spelling mistakes and wrong pluralization, since all of the terms were originally presented in singular form (e.g. spoons or sppoon were corrected to spoon, if and only if the mistake or pluralization was obvious and did not alter the meaning of the word).

The statistical analysis was conducted using the open source software R (version 1.0.136). Table 2 gives an overview of the means of the relevant results. Note that as mentioned earlier, the activity is also part of the results (head rotation, walking distance and time spent in the VMP). The rotation was measured as the accumulated degree of head rotation during the memorization phase in the VMPs.

\section{Table 2. Overview}

\begin{tabular}{|r|r|r|}
\cline { 2 - 3 } \multicolumn{1}{c|}{} & $\begin{array}{r}\text { Group 1 } \\
\text { (image-text) }\end{array}$ & $\begin{array}{r}\text { Group 2 } \\
\text { (text-only) }\end{array}$ \\
\hline N & 23 & 28 \\
\hline Strict Score & 0.3109 & 0.1107 \\
\hline Lenient Score & 0.7924 & 0.6759 \\
\hline Time (s) & 694.1 & 709.2 \\
\hline Rotation & 9316 & 6241 \\
\hline Meters & 277.9 & 345.2 \\
\hline
\end{tabular}

The evaluation of the hypotheses $\mathrm{H} 1$ and $\mathrm{H} 2$ was performed by analyzing the central tendencies in the data samples of group one (image-text) and two (textonly). First, the histograms of the distribution of the strict and lenient scores in both groups were considered. Significant results in the Shapiro-Wilk test confirm that all four data samples are very likely nonnormally distributed, so the p-values for the strict score are 0.0017 (group 1: image-text) and 0.0001 (group 2: text-only). The p-values for the lenient score are 0.0253 (group 1: image-text) and 0.0084 (group 2: text-only).

Due to the non-normal distributed data, both hypotheses were evaluated by two-sided MannWhitney $U$ tests. A significance level below 0.1 was considered as a trend effect, while values below 0.5 and 0.1 were seen as significant (similar to Legge et al. (2012)). The mean value of the strict score of group 1 (image-text) is 0.31 and the mean value of group 2 
(text-only) is 0.11 . Results of the Mann-Whitney U test show a significant difference on a level below 0.01 ( $p$ value $=0.008)$. The means of the lenient scores are 0.79 (group 1: image-text) and 0.68 (group 2: text-only). Again, the difference is significant on a level below 0.05 (p-value $=0.029)$.

The data shows that the image-text group achieved higher scores than the text-only group. The difference between the lenient scores is about $11 \%$ and is approx. 1.17 times higher in the first group. The strict score in the first group is approx. 2.81 times higher than in the second group (difference approx. 20\%).

Both tests showed significant differences between the central tendencies of the strict and lenient scores (at least $\mathrm{p}<0.05)$. Consequently, the data supports the hypotheses $\mathrm{H} 1$ and $\mathrm{H} 2$.

As mentioned in the description of the procedure, the prototypes also measured the time (in seconds) that the participants spent in the VMP. This analysis is intended to ensure that the results are not skewed by a group that spent significantly more or less time in the VMP. Again, histograms and Shapiro-Wilk tests were considered and indicated the time being normally distributed (group 1: $\mathrm{p}=0.8477$, group 2: $\mathrm{p}=0.1802$ ). Therefore, Welch's t-test was performed to reveal possible differences of means (group 1: 649.1s, group 2: 709.2s). This t-test is robust against unequal sample sizes $[35,38]$. Results indicate no significant difference between the groups $(\mathrm{df}=48.78, \mathrm{p}=0.8091)$. Hence, the time the participants spent in their prototypes did not differ on a significant level. Therefore, the time is not considered as an independent factor that influenced the difference of the strict and lenient scores in this case.

Another noteworthy effect was identified performing an analysis of correlation (Pearson). In contrast to the image-text group, the lenient and strict scores of the text-only group correlate positively with the intensity of the users' head rotation (level 0.4 , $\mathrm{p}<.05)$. This might support the idea behind the external vMOL. More precisely, the text-only treatment probably requires more activity in the sense of searching an adequate locus by moving around the head. Hence, a positive correlation between activity and the memorization accuracy possibly predicts the compliance of the participants to the MOL protocol, especially in a text-only treatment.

\section{Discussion}

As seen in the previous section, the data analysis supports the hypotheses $\mathrm{H} 1$ and $\mathrm{H} 2$. This indicates that the combination of images and text also leverages the memorization accuracy in the context of a VMP. The difference of the lenient scores $(11 \%)$ between the groups is certainly noteworthy. However, the strict score improved more clearly by the factor 2.81 . This effect might be explained by the design of the prototypes. Since the image-text VMP had fixed loci, the text-only participants had to choose the loci on their own. This design gave the students the opportunity to create their loci in a manner that did not follow a certain order if one walks mentally through the VMP. Nevertheless, subjects were instructed to memorize the items in order and the analysis implies a superior effectiveness of the image-text concept over the text-only VMP.

\subsection{Limitations \& Future Research}

At this point it is important to note that future studies could refine the experimental design and reduce the difference of the treatments between the two experimental groups in order to evaluate the users' interaction with the loci in a more detailed manner. For instance, putting the text-only items at the same places as the image-text items would align the presentation of the loci between the experimental groups. Alternatively, one could present the image-text loci at the center of the screen and therefore without a fixed position in the virtual surrounding. Hence, the process of associating the item with a certain place would happen completely in the user's mind in both settings. These modifications would reduce the difference of the treatments between the two experimental settings. In the first case, both groups would have a predefined route and in the second case both groups could freely choose their preferred path of loci. Furthermore, one could implement a feature that allows the participants to select the place where $\mathrm{s} /$ he wants to put the items. This way, the act of self-choosing the exact location, which is a central part of the traditional MOL, would be enabled in both groups as well.

So, future prototypes and studies should evaluate explicitly how the loci design and the degree of interaction helps the user to memorize the to-beremembered items. As shown in section two, a variety of different loci have already been implemented, integrated in the virtual world and enriched with other media features like sound, animations or interactivity. It is necessary to evaluate how cognitive theories can help to find design principles for highly memorable loci for VMPs.

This study was mainly conducted with technically oriented students in their mid-twenties. Therefore, further investigations would certainly profit from a wider range of professions and age.

The factors imaginativeness and spatial sense might be relevant independent variables when participants have to use their creativity to create mental 
representations of the loci in a spatial context. Measuring and analyzing these factors in a VMP setting could reveal relevant insights for the potential target group and future VMP concepts.

Referring to Legge et al. (2012), they suggested to find appropriate ways to measure the compliance of the participants with the vMOL protocol. This approach shall also be outlined at this point. Since the compliance is an important factor when it comes to interpreting the analysis results, unfortunately a sophisticated method for the measurement is yet to be developed.

\subsection{Conclusion}

The results imply that the dual coding theory also applies in the context of a VMP. It could be shown, that referring to earlier studies, the concept of visualized loci outperforms the text-only loci for the memorization process. Hence, in a next step it would be interesting to evaluate other, more complex types of loci, enriched with multimedia cues, animations or interactive behavior. Also, different displays with different levels of immersion could be compared to each other in order to reveal the impact of the factor immersion on the users' memorization process in a vMOL protocol.

\section{References}

[1] Agarwal, R. and Karahanna, E. Time Flies When You're Having Fun: Cognitive Absorption and Beliefs about Information Technology Usage. MISQ 24, 4 (2000), 665.

[2] Bellezza, F.S. Mnemonic devices: Classification, characteristics, and criteria. Review of Educational Research 51, 2 (1981), 247-275.

[3] Bredl, K., Groß, A., Hünniger, J., and Fleischer, J. The Avatar as a Knowledge worker? How immersive 3D virtual environments may foster knowledge acquisition. Leading Issues in Knowledge Management, Volume Two 2, (2012), 222.

[4] Brehmer, Y., Li, S.-C., Mueller, V., von Oertzen, T., and Lindenberger, U. Memory plasticity across the life span: Uncovering children's latent potential. Developmental Psychology 43, 2 (2007), 465-478.

[5] Dede, C. Immersive Interfaces for Engagement and Learning. Science 323, 5910 (2009), 66-69.

[6] Dresler, M., Shirer, W.R., Konrad, B.N., et al. Mnemonic Training Reshapes Brain Networks to Support Superior Memory. Neuron 93, 5 (2017), 1227-1235.e6.
[7] Fassbender, E. and Heiden, W. The virtual memory palace. Journal of Computational Information Systems 2, 1 (2006), 457-464.

[8] Fleming, M.L. On pictures in educational research. Instructional Science 8, 3 (1979), 235-251.

[9] Hartwig, M.K. and Dunlosky, J. Study strategies of college students. Psych. Bulletin\&Review 19, 1 (2012), 126134.

[10] Hedman, A. and Bäckström, P. Rediscovering the Art of Memory in Computer Based Learning-An Example Application. (2000).

[11] Hettinger, L.J. and Riccio, G.E. Visually induced motion sickness in virtual environments. Presence: Teleoperators \& Virtual Environments 1, 3 (1992), 306-310.

[12] Hevner, A.R., March, S.T., and Ram, S. Design Science In Information Systems Research. MIS Quarterly 28, 1 (2004), 75-105.

[13] Jund, T., Capobianco, A., and Larue, F. Impact of Frame of Reference on Memorization in Virtual Environments. IEEE (2016), 533-537.

[14] Kanske, P. and Kotz, S.A. Leipzig Affective Norms for German: A reliability study. Behavior Research Methods 42, 4 (2010), 987-991.

[15] Krathwohl, D.R. A Revision of Bloom's Taxonomy: An Overview. Theory Into Practice 41, 4 (2002), 212-218.

[16] Legge, E.L.G., Madan, C.R., Ng, E.T., and Caplan, J.B. Building a memory palace in minutes. Acta Psychologica 141, 3 (2012), 380-390.

[17] Levin, J.R. and Levin, M.E. Scientific Mnemonomies: Methods for Maximizing More Than Memory. American Educational Research Journal 27, 2 (1990), 301-321.

[18] Lin, J.J.-W., Duh, H.B., Parker, D.E., Abi-Rached, H., and Furness, T.A. Effects of field of view on presence, enjoyment, memory, and simulator sickness in a virtual environment. Virtual Reality, 2002. Proceedings. IEEE, IEEE (2002), 164-171.

[19] Liu, L., Ip, R., Shum, A., and Wagner, C. Learning Effects of Virtual Game Worlds: An Empirical Investigation of Immersion, Enjoyment and Performance. (2014).

[20] Maguire, E.A., Valentine, E.R., Wilding, J.M., and Kapur, N. Routes to remembering: the brains behind superior memory. Nature Neuroscience 6, 1 (2002), 90-95.

[21] Malaga, R.A. The effect of stimulus modes and associative distance in individual creativity support systems. Decision Support Systems 29, 2 (2000), 125-141. 
[22] Mania, K. and Chalmers, A. The effects of levels of immersion on memory and presence in virtual environments: A reality centered approach. CyberPsychology \& Behavior 4, 2 (2001), 247-264.

[23] Mann, J., Polys, N., Diana, R., Ananth, M., Herald, B., and Platel, S. Virginia tech's study hall: A virtual method of loci mnemotechnic study using a neurologically-based, mechanism-driven, approach to immersive learning research. Virtual Reality (VR), 2017 IEEE, IEEE (2017), 383-384.

[24] Martín-Gutiérrez, J. Virtual Technologies Trends in Education. EURASIA Journal of Mathematics, Science and Technology Education 13, 1 (2017).

[25] Mayer, R.E. and Moreno, R. Nine Ways to Reduce Cognitive Load in Multimedia Learning. Educational Psychologist 38, 1 (2003), 43-52.

[26] McCabe, J. Metacognitive awareness of learning strategies in undergraduates. Memory \& Cognition 39, 3 (2011), 462-476.

[27] Paivio, A. The Empirical Case For Dual Coding. In Imagery, Memory and Cognition. 1983, 307-332.

[28] Paivio, A. and Lambert, W. Dual coding and bilingual memory. Journal of verbal learning and verbal behavior 20,5 (1981), 532-539.

[29] Peffers, K., Tuunanen, T., Rothenberger, M.A., and Chatterjee, S. A Design Science Research Methodology for Information Systems Research. Journal of Management Information Systems 24, 3 (2007), 45-77.

[30] Putnam, A.L. Mnemonics in education: Current research and applications. Translational Issues in Psychological Science 1, 2 (2015), 130-139.

[31] Ragan, E.D., Bowman, D.A., and Huber, K.J. Supporting cognitive processing with spatial information presentations in virtual environments. Virtual Reality 16, 4 (2012), 301-314.

[32] Ragan, E.D., Sowndararajan, A., Kopper, R., and Bowman, D.A. The Effects of Higher Levels of Immersion on Procedure Memorization Performance and Implications for Educational Virtual Environments. Presence:

Teleoperators and Virtual Environments 19, 6 (2010), $527-$ 543.

[33] Roediger, H.L. The effectiveness of four mnemonics in ordering recall. Journal of Experimental Psychology: Human Learning and Memory 6, 5 (1980), 558.

[34] Ross, J. and Lawrence, K.A. Some observations on memory artifice. Psychonomic Science 13, 2 (1968), 107108.

[35] Ruxton, G.D. The unequal variance t-test is an underused alternative to Student's t-test and the MannWhitney U test. Behavioral Ecology 17, 4 (2006), 688-690.

[36] Shepard, R.N. Recognition memory for words, sentences, and pictures. Journal of verbal Learning and verbal Behavior 6, 1 (1967), 156-163.

[37] Standing, L. Learning 10000 pictures. Quarterly Journal of Experimental Psychology 25, 2 (1973), 207-222.

[38] Welch, B.L. The generalization of "Student's" problem when several different population variances are involved. Biometrika 34, 1-2 (1947), 28-35.

[39] Witmer, B.G. and Singer, M.J. Measuring Presence in Virtual Environments: A Presence Questionnaire. Presence 7, 3 (1998), 225-240.

[40] Wong, J. and Storkerson, P. Hypertext and the Art of Memory. Visible Language 31, 2 (1997), 126-157.

[41] Yates, F.A. and Yates, F.A. The art of memory. Routledge, London; New York, 1999. 